\title{
ROLE OF MONOTONOUS ATTENTION IN TRAFFIC VIOLATIONS, ERRORS, AND ACCIDENTS
}

\author{
Nebi Sümer*, Belgin Ayvaşık*, Nurhan Er**, and Türker Özkan* \\ *Department of Psychology, Middle East Technical University, Ankara -Turkey \\ **Department of Psychology, Ankara University, Ankara -Turkey \\ E-mail: nsumer@metu.edu.tr
}

\begin{abstract}
Summary: Recent traffic Law in Turkey requires that drivers whose driving licenses were withheld because of their serious traffic offences be subjected to "psychotechnical assessment" tests. These tests must include measures for psychomotor and mental abilities such as monotonous attention, peripheral perception, and reasoning. Currently, we are in process of developing a computer based psychotechnical driver test system. This study investigates the validity of the monotonous attention test that is one of the measures in our system. Participants were 79 volunteer drivers from Ankara, Turkey. First, drivers completed the Driver Behavior Questionnaire (DBQ) measuring violations and errors and demographic items regarding drivers' history of offences and accidents. Second, participants were asked to respond the Traffic Monotonous Attention Test that is a cancellation task. Initial analysis revealed that the correlations between the scores of attention index and other major variables were not significant. Therefore, a tripartite split was performed on the participants' attention scores. The results of ANOVAs revealed that those who had medium level of attention (incorrect responses) reported higher levels of driving errors than both high and low attention groups. The examination of the relationships between accident type and the continuous attention scores indicated that those having active accidents also had higher levels of both incorrect and omitted responses than no accident and passive accident groups. Findings of this study seem to imply that individual differences in the monotonous attention should be related with accident involvement and therefore, should be considered in devising a computer-based system including the measure of monotonous attention.
\end{abstract}

Compared to the other European countries, Turkey has a relatively higher rate of traffic accidents causing a large number of fatalities and injuries. Every year about five thousand people are killed, and about one hundred thousand of people are injured in road traffic accidents in Turkey. These accidents also result in a substantial amount of cost to Turkish economy. Considering this situation, recent traffic Law in Turkey requires that drivers whose driving license was withheld because of their serious traffic offences be subjected to "psychotechnical assessment" tests. Psychotechnical assessment techniques consist of computer-based tests including psychomotor and mental abilities such as monotonous attention, peripheral perception, and reasoning. The purpose of the assessment is to identify drivers whose cognitive and psychomotor abilities are suitable for driving.

Attention processes (e.g., monotonous, selective and divided) are one of the most important information processing abilities, which are related to driving behavior. Past studies have yielded high correlations between accident rates, vehicle speed and driver's attention to the complex and 
rich stimuli in the traffic environment (Arthur, Strong, and Williamson, 1994; Kahneman, BenIshai, and Lotan, 1973; Robinson, 1975).

In general, attention has been defined as the selection of some subset of possible information among variety of different stimuli, either external or internal, for further processing which requires a state of concentration on certain stimuli (Leahey, and Harris, 1997). In traffic situations, monotonous, selective, and divided attention are necessary to able to process multiple stimuli. Drivers are exposed to various stimuli from different modalities (e.g., visual and auditory) and have to attend and perceive them to pursue their driving behavior safely. Traffic environment also provides a complex information-processing situation for drivers in terms of direction, continuity, quantity, quality, and ambiguity of stimulus. The degree of attention processes would be interacted with the complexity of stimuli in traffic environment.

Traditionally, attention tasks consist of a target stimulus and distractor stimuli in different modalities. Distractors are usually similar to the target although they are not exactly the same. The participants need to select the target stimulus among the distractors and then respond to the target under focused or divided conditions (Posner, 1986; Schnider and Shiffrin, 1977).

Currently, we are in process of developing a computer-based psychotechnical assessment system for drivers including a measure for monotonous attention named as the Traffic Monotonous Attention Test (TMAT) for Turkish drivers. The TMAT is a cancellation task developed on the basis of Mesulam's cancellation tasks (1985). The main purpose of this study was to investigate the validity of the TMAT in a paper-pencil format before computerized version. For this purpose, we specifically examined the relationship between monotonous attention and the number and type of accidents in last three years and as well as other traffic related behaviors such as traffic offences and overtaking tendency. Following the suggestion of Elander, West and French (1993), we classified accidents as active (i.e., a driver hits another object, person or vehicle) and passive (i.e., a driver is hit by another driver) in terms of the role of the drivers in the accidents. Passive accidents are mostly related to the driver's annual mileage although active accidents seem to be an important indicator of driver's accident liability (Elander, West and French, 1993; Lajunen, 1999). We particularly hypothesized that (1) low levels of monotonous attention are expected to be related with high levels of accident involvement, errors, and violations, and (2) those who have relatively more active accidents are more likely to have low levels of monotonous attention than those who have passive accidents.

\section{Method}

\section{Participants}

Participants were 79 volunteer drivers (51 male and 28 female) from Ankara, Turkey. Of the participants, 58 were automobile drivers and 21 were taxi and service drivers. The mean age of the participants was 30 years $(\mathrm{SD}=9.84)$ and average annual mileage driven was $19.598 \mathrm{~km}$.

\section{Measures and Procedures}

Participants were asked to complete following questionnaires and to respond to a monotonous attention task. 
Traffic Monotonous Attention Test (TMAT) is a paper and pencil version of computer based Turkish Psychotechnical Assessment System developed by the authors. As a fraction of the test was depicted in Figure 1, the TMAT is a cancellation task consisting of differently designed versions of a road traffic sign arranged in a 15X21 matrix. The task was to identify 75 target items randomly distributed within the matrix. Participants were completed the task in a silent room and they were given a maximum of five minutes to complete the TMAT. The mean completion time was 190 seconds (range 80 - 300 seconds, and SD = 48.31). Correct, incorrect and omitted responses were calculated for each participant. The average correct responses were 58.89 (range 30 - 74 items, $\mathrm{SD}=11.85$ ), the average incorrect responses were 6.22 (range 0 - 30 items, $S D=5.65$ ), and for omitted items were 16.20 (range 1 - 45, SD = 11.92). Initially, a total score for correct, incorrect, and omitted items were calculated, and then, a standard score for participants was obtained by dividing the number of incorrect responses to correct responses multiplied by 100 . Thus, a percentage score was calculated indicating the rate incorrect responses to correct ones.

\section{(1)}

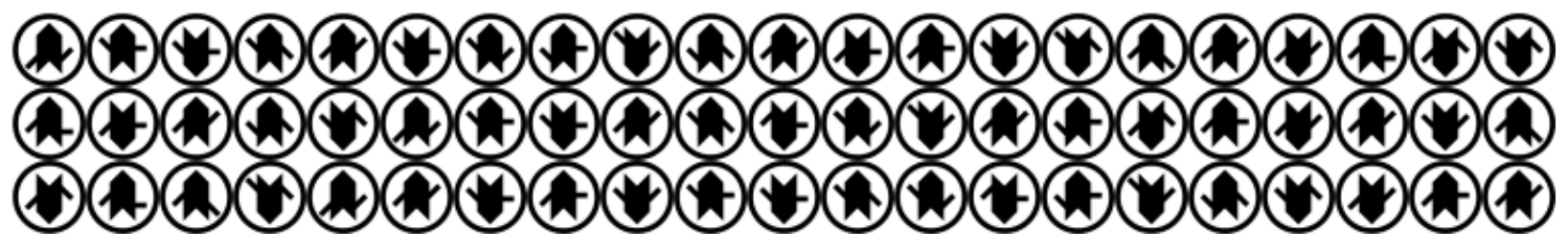

Figure 1. A fraction from the TMAT.

A 28 item measure of Driver Behavior Questionnaire ( $D B Q$ ) which was originally developed by Reason, Manstead, Stradling, Baxter \& Campbell (1990) and translated by Sumer (2000) was used to measure violations and errors in driving. Items measuring violations and errors only were included in this study and items measuring lapses were excluded. We also added 9 items developed by Sumer (2000), which measure violations and errors commonly seen among Turkish drivers. Participants were responded to the DBQ with 4-point scales. Earlier factor analyses with orthogonal rotation yielded two factors corresponding the violations and errors. Two subscales were constructed on the basis of factor analysis. The violation subscale consisted of 17 items and the errors subscales included 11 items and the scales revealed satisfactory internal consistencies (alpha $=.84$ and .83 , respectively) (Sumer, 2000). In this study, alpha coefficients were .82 for the violation subscale and .73 for the errors subscale.

Participants also completed a demographic questionnaire about their traffic accident history and roles in these accidents, traffic violation or offence tickets taken regarding the last three years of their driving experiences, type of accidents (active vs. passive), and their driving experiences such as average speed they drive in intercity motorways and within city roads.

\section{Results}

As seen in the Table 1, the intercorrelations among the major variables of the study indicated attention index was positively and significantly correlated with the omitted items $(\underline{\mathrm{r}}=.32, \mathrm{p}<$ $.01)$. 
Table 1. Intercorrelations among the major variables of the study

\begin{tabular}{lcccccccc}
\hline Variables & 1 & 2 & 3 & 4 & 5 & 6 & 7 & 8 \\
\hline 1. Attention Index & - & & & & & & & \\
\hline 2. Omitted items & $.32^{* *}$ & - & & & & & & \\
\hline 3. Errors & .13 & -.01 & - & & & & \\
4. Violations & $.20^{+}$ & .03 & $.66^{* *}$ & - & & & \\
5. Number of acc. & .10 & .07 & $.23^{*}$ & $.33^{* *}$ & - & & \\
6. Total tickets taken & .02 & .13 & .11 & .18 & $.31^{* *}$ & - & & \\
7. Speed on motorway & $-.25^{*}$ & -.15 & $.36^{* *}$ & $.46^{* *}$ & $.20^{+}$ & $.31^{* *}$ & - & \\
8. Speed in city roads & .05 & -.02 & $.37^{* *}$ & $.55^{* *}$ & .11 & $.27^{*}$ & $.57^{* *}$ & - \\
9. Overtaking rates & .06 & .12 & .15 & $.52^{* *}$ & $.21^{+}$ & $.39^{* *}$ & $.35^{* *}$ & $.32^{* *}$ \\
\hline
\end{tabular}
${ }^{+} \mathrm{p}<.10 * \mathrm{p}<.05, * *<.01$

Regarding the major variables of the study, the attention index was marginally correlated with violations only $(\underline{r}=.20, \underline{p}<.10)$ and this was not significantly correlated with errors, violations, number of accidents and other major variables although their relationships were in expected direction. The number of omitted items did not correlate with other variables. However, as expected, traffic errors and violations were significantly correlated the number of accidents involved, ticket taken in the last three years, and the average speed in both city roads and motorways, tendency for overtaking.

Given the fact that the relationship between monotonous attention and other variables may be nonlinear, in next series of analyses, we categorized participants into three groups as high, medium, and low on the basis of their score on attention index and conducted a series of one-way ANOVAs with these three attention groups on the major variables followed by Students' Newman-Keuls post-hoc analysis. As seen in Table 2, attention levels had an significant effect on the traffic errors only $(\underline{F}(2,75)=3.62, \underline{p}<.05)$. As illustrated in Figure 2 , post-hoc analysis revealed that those who had medium level of attention index score (incorrect responses) reported higher levels of traffic errors than both high and low attention groups. The differences among the three groups were not statistically significant on other variables.

Table 2. Means. standard deviations and F values of variables in the study

\begin{tabular}{lcccccccc}
\hline \multicolumn{7}{c}{ High } & \multicolumn{7}{c}{ Medium } & Low & F \\
\hline Variables & Mean & Std. & Mean & Std. & Mean & Std. \\
\hline Errors & 1.26 & .16 & 1.44 & .38 & 1.25 & .17 & $3.62^{*}$ \\
Violations & 1.60 & .31 & 1.67 & .54 & 1.70 & .45 & .185 \\
Number of accidents & .85 & 1.17 & .95 & 1.12 & 1.00 & 1.25 & .068 \\
Total offences & 1.14 & 1.45 & .87 & .99 & .80 & 1.19 & .329 \\
Speed on motorway & 111 & 19 & 109 & 32 & 98 & 34 & 1.13 \\
Speed in city roads & 69 & 15 & 64 & 19 & 69 & 17 & .406 \\
Overtaking rates & 1.77 & .89 & 1.54 & .77 & 2.00 & .85 & 1.63 \\
\hline$*<.05$ & & & & & & &
\end{tabular}




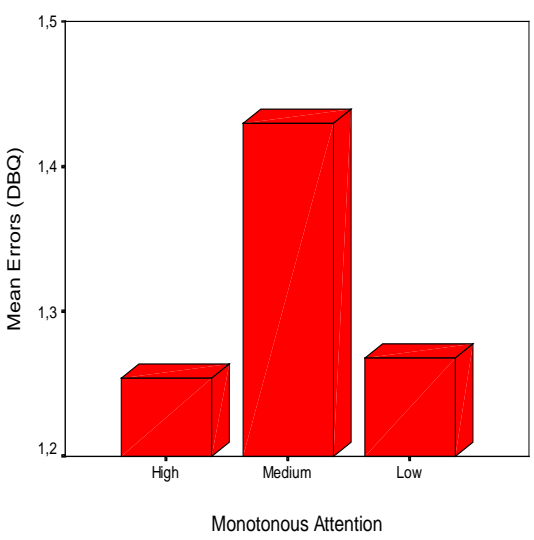

Figure 2. Mean errors by the three attention groups

We examined the relationships between accident type and monotonous attention by creating three groups of drivers who have (1) no accidents, (1) active accidents, and (3) passive accidents. As depicted in Figure 3, the results of the ANOVA indicated that those who active accidents had higher mean $(\underline{\mathrm{M}}=17.01)$ from attention index (incorrect response) than both no accident $(\underline{\mathrm{M}}$ $=9.44)$ and passive accident groups $(\underline{\mathrm{M}}=6.82)(\mathrm{F}(2,75)=5.24, \underline{\mathrm{p}}<.01)$. No accident and passive accident groups did not statistically differ from each other. A final ANOVA was run on the mean omitted items. The type of accidents had a marginally significant effect on the omitted attention items (only $(\underline{F}(2,75)=2.63, \underline{p}<.07)$. As shown in the Figure 4, consistent with the previous analyses, the active accident group had also higher omitted attention items $(X=21.00)$ than both nonaccident group $(\underline{\mathrm{M}}=14.15)$ and passive accident group $(\underline{\mathrm{M}}=14.15)$.

\section{Discussion}

Results from this study indicated that monotonous attention is related to the type of accidents and traffic errors. Those who involve in active type of accidents possessed lower levels of monotonous attention, and they also omitted more items than those who had no accidents and those who had passive accidents only. To our knowledge, there was no similar study examining the relationship between accident type and attention. However, supporting our findings, Avolio, Kroeck, and Panek (1985) found differences between nonaccident and accident groups on a series of attention task. Findings of this study seem to imply that individual differences in

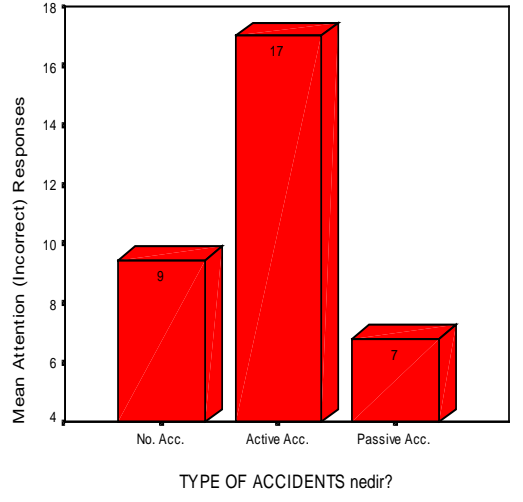

Figure 3. Mean incorrect responses by the type of accidents

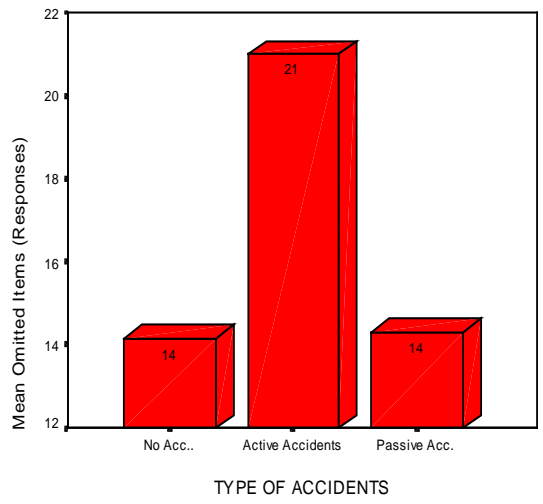

Figure 4. Mean omitted responses by the type of accidents 
monotonous attention may be related to active accident risk, rather than a general accident involvement. Although monotonous attention indices used in this study were not significantly correlated with driving errors, overtaking tendency and other major variables, the marginally significant correlation between monotonous attention and violation seems to suggest that inadequacy in attention as an information processing factor may distract drivers in obeying traffic rules. It can be concluded that active accident repeaters should be checked for their adequacy in attention processes. This also gives a chance to this measure for applying in selection purposes.

Unexpectedly, we found that there is a nonlinear relationship between driving errors and monotonous attention. As depicted in Figure 2, those who have intermediate level of monotonous attention reported significantly higher levels of errors measured by the DBQ than both those have low and high levels of attention. This may be interpreted that those who have low levels of attention may be aware of this and act really cautiously in traffic situation and thus, avoid errors. Those who have high levels of monotonous attention can benefit from their ability to detect possible hazards in traffic situations. Those who have intermediate level of monotonous attention, however, may not be able to assess their relative limitation which may cause an "illusive over-confidence" about their cognitive abilities associated with driving, and in turn, this may negatively influence their vigilance causing high rates of errors.

Finally, we should be cautious in interpreting the results of this study because of certain limitations. First, assessment of time is a problem in paper-pencil tasks which may be directly related with participants' actual performance. In future studies, computerized version of the test should be utilized. We use a limited and mixed sample (both automobile and taxi drivers) that may confound our findings. Further studies should examine the role of monotonous attention in both driving skills and styles by considering above limitations. 


\section{REFERENCES}

Avolio, B. J., Kroeck, K. G., \& Panek, P. E. (1985). Individual differences in information processing ability as a predictor of motor vehicle accidents. Human Factors, 27 (5), 577-587.

Arthur, W., Strong, M.H., \& Williamson, J. (1994). Validation of a visual attention test as a predictor of driving accident involvement. Journal of Occupational and Organizational Psychology, 67,173-182.

Elander, J., West, R., \& French, D. (1993). Behavioral correlates of individual differences in road traffic crash risk: An examination of methods and findings. Psychological Bulletin, 113, 269-294.

Kahneman, D., Ben-Ishai, R., \& Lotan, M. (1973). Relation of a test of attention to road accidents. Journal of Applied Psychology, 58, 113-115.

Lajunen, T. (1999). The role of personality characteristics in traffic accident liability: research findings and methodological considerations. Türk Psikoloji Yazıları, 2(4), 97-107.

Leahey, T.H., \& Harris, R.J. (1997). Learning and cognition ( $4^{\text {th }}$ ed.). New Jersey: Prentice Hall.

Mesulam, M. (1985). Principles of behavioral neurology. Philadelphia: F.A. Davis.

Posner, M. I. (1986). Chronometric explorations of mind. New York: Oxford University Press.

Reason, J., Manstead, A., Stradling, S., Baxter, J., \& Campbell, K. (1990). Errors and violations on the roads. Ergonomics, 33, 1315-1332.

Robinson, G.H. (1975). Toward measurement of attention as a function of risk and risk preference in man-machine systems. Human Factors, 17(3), 236-242.

Schneider, W., \& Shiffrin, R.M. (1977). Controlled and automatic human information processing: I. Detection, search, and attention. Psychological Review, 84, 1-66.

Sümer, N. "The role of personality in behaviors of accident prone drivers” (Report No: AFP-99.01.04.03). Ankara: Middle East Technical University, 2000. 